\title{
Fenomenología
}

\section{Figura del padre en relación a la crianza: pasado, presente y futuro.}

\section{Figure of the father in relating to the upbringing: past, present and future}

\section{A figura do pai em matéria de educação: passado, presente e futuro.}

Ricardo López Marín ${ }^{1}$, Alonso Molina Rodríguez ${ }^{1}$, María Emilia Martínez Roche², Davinia Frutos Molina², Teresa Molina Rodríguez ${ }^{3}$.

${ }^{1}$ Residente de Matrón de $2^{\circ}$ año de la Unidad docente de Matronas de la Universidad de Murcia y del Servicio Murciano de Salud.

${ }^{2}$ Coordinadora de la Unidad docente de Matronas de la Universidad de Murcia y del Servicio Murciano de Salud.

${ }^{3}$ Enfermera por la Universidad de Murcia.

Cómo citar este artículo en edición digital: López Marín R; Molina Rodríguez, A; Martínez Roche MaEF; Frutos Molina, D; Molina Rodríguez T.(2012) Figura del padre en relación a la crianza: pasado, presente y futuro. Cultura de los Cuidados. (Edición digital) 16, 32. Disponible en: <http://dx.doi.org/10.7184/cuid.2012.32.02>

Correspondencia: Ricardo López Marín.Calle Telefonista Encarna Ayala, n.2 1 1C. 30570 Beniaján Murcia. 626129485 ricardo_ lopez_marin@hotmail.com

Recibido: 20/10/2011 - Aceptado: 17/12/2011

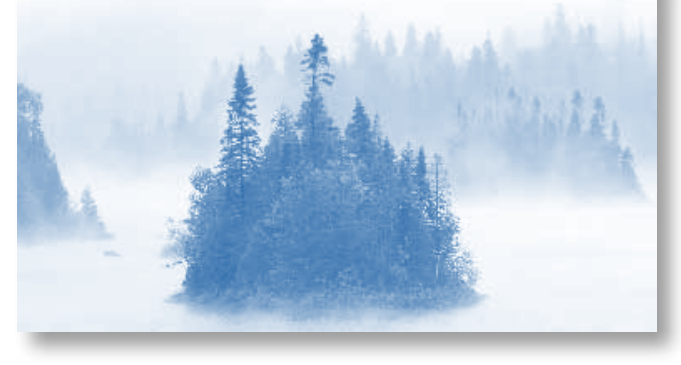

ABSTRACT

The current situation in the West reflects a gradual change of the father in the upbringing of their offspring, as reflected in its theory Madeleine Leininger, one must know the characteristics of this phenomenon to implement culturally congruent care. Based on the concept of Mercer "roles are not fixed, so no matter which person is responsible for performing the different roles" (Marriner, 2007) can mimic the maternalpaternal-filial subsidiary.

We propose a qualitative research based on in-depth interview in order to study: parents (men) novice, and discussion groups professional midwives as the most representative figure and is directly involved with women's reproductive health and care the newborn.

Thus we can say that the typical family idea is accepted as is in crisis around the nineties, there emerges the figure of the new parents, a concept introduced by Bonino (2003). We met a father whose presence in the care of children is of great importance in the proper development of these (Alberdi \& Escario, 2007).

Therefore we must work on the model to define the New Parent involvement in raising it. The midwife is the professional most qualified to serve as agents of change.

Key words: Parenting, Father, Midwife.

\section{RESUMO}

A situação atual no Ocidente reflete uma mudança gradual do pai na educação dos seus filhos, como refletido na sua teoria Madeleine Leininger, é preciso conhecer as características 
deste fenômeno para implementar cuidados culturalmente congruentes. Baseado no conceito de Mercer "papéis não são fixos, então não importa onde a pessoa é responsável por executar os diferentes papéis" (Marriner, 2007) podem imitar a subsidiária maternopaterno-filial.

Propomos uma pesquisa qualitativa com base em entrevistas em profundidade a fim de estudar: os pais (homens) novato, e grupos de discussão parteiras profissionais como a figura mais representativa e está diretamente envolvido com a saúde reprodutiva das mulheres e cuidados o recém-nascido.

Assim, podemos dizer que a idéia típica família é aceito como está em crise em torno da década de noventa, emerge a figura de os novos pais, um conceito introduzido por Bonino (2003). Encontramos um pai cuja presença no cuidado das crianças é de grande importância no desenvolvimento adequado desses (Alberdi \& Escario, 2007).

Portanto, devemos trabalhar com o modelo para definir o envolvimento dos pais em Nova elevando-o. A parteira é o profissional mais qualificado para servir como agentes de mudança.

Palavras-chave: Parenting, Pai, matronal.

\section{RESUMEN}

La situación actual en el mundo occidental refleja un cambio gradual de la figura del padre en la crianza de su descendencia; según refleja Madeleine Leininger en su teoría, debemos conocer las características de este fenómeno para aplicar cuidados culturalmente congruentes. Basándonos en el concepto de Mercer "los roles no están fijados; por tanto, carece de importancia qué persona se encarga de realizar los distintos roles" (Marriner, 2007) podemos asemejar el vínculo materno-filial al paterno-filial.

Proponemos una investigación de corte cualitativo basada en la entrevista en profundidad al objeto de estudio: padres (hombres) noveles; y grupos de discusión a matronas como figura profesional más representativa y que está directamente implicada con la salud reproductiva de la mujer y del cuidado del recién nacido.

Así podemos afirmar que la idea de familia típica tal y como es aceptada entra en crisis entorno a los años noventa, emergiendo así la figura de los Nuevos Padres, concepto introducido por Bonino (2003). Nos encontramos con un padre cuya presencia en el cuidado de los hijos tiene una gran importancia en el buen desarrollo de los mismos (Alberdi \& Escario, 2007).

Por tanto debemos trabajar sobre el modelo de Nuevos Padres para definir la implicación de éste en la crianza. La matrona es la profesional más cualificada para servir como agente impulsor de cambio.

Palabras clave: Crianza, padre, matrona.

\section{INTRODUCCIÓN}

\section{Estado de la cuestión. Marco Teórico}

El concepto de paternidad ha sido en las últimas décadas un término cambiante. Tanto es así que la sociedad ha observado una mutabilidad del rol del hombre en el ámbito familiar. Existe una controversia acerca del aporte del hombre a la familia.

Si entendemos la paternidad tal y como es definida por la Real Academia de la Lengua Española (2001) "Cualidad de padre", respecto al término padre esta misma entidad la define como "Varón o macho que ha engendrado". De dichas definiciones, observamos que no impli- 


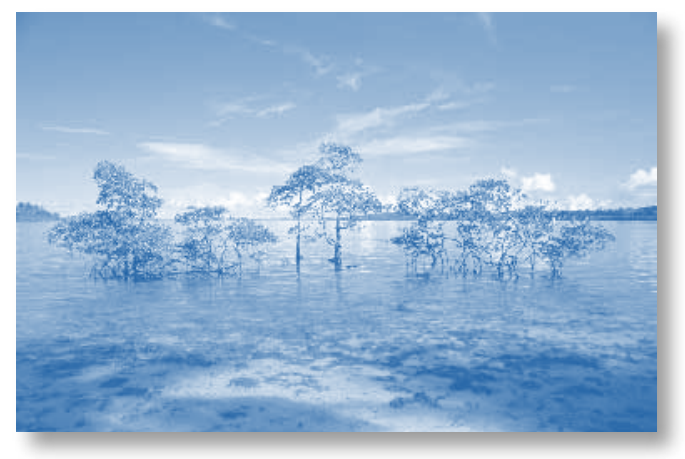

ca rol alguno con respecto a la familia.

No obstante, la realidad es bastante diferente, como encontramos en Vicuña y Reyes (2002)

"Sandra Ferketich descubrió que la tendencia a criar se encuentra tanto en varones como en mujeres, así como el deseo de sentirse conectado emocionalmente con los hijos. Otro investigador, Ross Parke, especifica que tanto los padres como las madres son igualmente capaces de interpretar las señales de sus hijos indicativas de hambre, molestias o fatigas, e igualmente capaces de responder a ellas de manera adecuada”. (p.1)

En nuestra construcción sobre la temática de la paternidad encontramos en la obra de $\mathrm{Pa}$ terna, Martínez y Rodes (2005) otros autores como

"Silverstein et al. (1999) que afirman que el rol paternal incluye, además de proveer de recursos económicos, el compromiso activo con la alimentación, cuidado y educación de los hijos, sin descuidar la parte afectiva y el estar en todo momento accesible emocionalmente. Por su parte, Lamb (1987) definió la conducta paternal a partir de los conceptos de accesibilidad, responsabilidad y obligación como compromiso. Asimismo en EEUU están surgiendo una serie de movimientos sociales que intentan reconstruir el rol de paternidad con el fin de incrementar la responsabilidad del padre, por ejemplo, a través de los movimientos de hombres pro-feministas (Levant \& Pollack, 1995) o los neoconservadores”. (p.275)

Como hemos visto la relación padre-hijo pasa del papel meramente protector hasta involucrase en la crianza en la sociedad actual. El punto de inflexión en la sociedad occidental fue probablemente en la década de los años 80 cuando hubo una inserción masiva de la mujer al mundo laboral y la solvencia económica de éstas.

Si abordamos la temática de la figura paternal desde el punto de vista de la Ciencia Enfermera, podemos utilizar la teoría de Ramona T. Mercer como sustento de nuestras afirmaciones. En su teoría primitiva titulada "Adopción del rol maternal", Mercer (1981) expone el concepto de adopción del rol maternal entendido como "proceso interactivo y de desarrollo que se produce a lo largo del tiempo en el cual la madre crea un vínculo con su hijo, aprende las tareas de cuidado del rol y expresa el placer y la gratificación con el rol”. En dicha definición entendemos que el cuidado de la madre hacia su hijo, está fundamentado en roles aprendidos. Mercer sostiene "los roles no están fijados; por tanto, carece de importancia que persona se encarga de realizar los distintos roles" (Mercer, 1990). Así el rol del padre cuidador puede compararse de forma idéntica al rol de madre cuidadora. Posteriormente la estudiosa realiza una revisión de su teoría y modifica su premisa, defendiendo que la crianza no es una mera cuestión de roles; desechó el término adopción del rol maternal para llegar al concepto de "Convertirse en madre”. Amplía la visión afirmando que algunos roles podrían terminar y convierte a la crianza en un compromiso de por vida (Marriner, 2009). En su modelo revisado habla de la consecución cronológica de cuatro etapas necesarias para convertirse en madre: 
1. "Compromiso y preparación.

2. Conocimiento, práctica y recuperación física.

3. Normalización.

4. Integración de la identidad materna.”

La etapa 1 hace referencia al embarazo en el que la mujer toma conciencia de los cambios y se prepara para la maternidad. La segunda habla de la adaptación y recuperación del embarazo y parto. En la tercera se produce una normalización y como última etapa tenemos la integración de la identidad materna tener una visión interiorizada de sí misma como madre (Mercer, 2004).

Si extrapolamos estos conceptos al campo de nuestro estudio, entendemos que tal y como Mercer los define son fácilmente semejables a la figura del padre en relación a la crianza, de manera que el padre podría pasar por dichas etapas hasta convertirse en padre de igual forma que lo hace la madre. Podemos afirmar que todas las etapas son análogas en la figura masculina (a excepción de la recuperación física de la fase 2 como es obvio).

Si continuamos con el estudio de las enfermeras teóricas encontramos que podemos enmarcar nuestra construcción sobre la paternidad en la teoría de los "Cuidados Culturales" de Madeleine Leininger, al tomar como centro de estudio la figura del padre en occidente para aplicar unos cuidados de calidad, ya que, los cuidados culturales son vitales para el bienestar, la salud, el crecimiento y la supervivencia (...) formando estos el medio holístico más amplio para saber, explicar, interpretar y predecir los fenómenos de los cuidados enfermeros (Leininger \& McFarland, 2005). Dándose solo estos cuando la enfermera tiene conocimiento y hace uso adecuado y coherente de los valores, expresiones y modelos.
Entendemos que para ofrecer unos cuidados culturalmente congruentes, según dice Marriner (2009) "no podemos separar las concepciones del mundo, la estructura social ni las creencias culturales cambiantes en nuestro medio" (p.481) como son la figura del padre en la cultura occidental actual y su función en la estructura social dentro y fuera de la familia. Siendo esencial el descubrimiento sistemático y creativo del conocimiento de este campo de estudio, que es relevante para entender el fenómeno que se nos presenta (Leininger, 1996).

Por todo ello se puede conseguir una reestructuración de los cuidados culturales, facilitando a los nuevos padres a reorganizar, cambiar y modificar en gran medida sus modos de vida para obtener nuevos resultados, diferentes y beneficiosos que respondan a las demandas de la sociedad actual en occidente.

\section{Justificación}

Nuestras competencias tal y como las definen la Confederación Internacional de Matronas (2005), especifican que la matrona "tiene una tarea importante en el asesoramiento (...), no sólo para la mujer, sino también en el seno de sus familias y de la comunidad. Este trabajo debe (...) extenderse a la salud de mujeres, la salud sexual o reproductiva, y el cuidado de los niños."

Asimismo, la situación actual en el mundo occidental refleja un cambio gradual de la figura del padre en la crianza de su descendencia, dándose una implicación mayor del papel paterno en esta, tal y como recoge Bonino (2003) "comienza a emerger un fenómeno que suele llamarse el renacimiento del padre o la aparición de los nuevos padres (...) alejada de los modelos de padre distante y autoritario" (p.173). Este fenómeno hace que surjan nuevas demandas tanto dentro del ámbito familiar 
y por extensión de la comunidad, haciéndose necesaria la actualización del corpus doctrinal entendiendo el cambio en toda su globalidad.

Nosotros como hombres profesionales de la matronería estamos especialmente motivados con el emergente cambio del rol paterno siendo necesario entender este fenómeno para dar un soporte adecuado planteándonos las siguientes cuestiones: ¿Cuál es la verdadera implicación del padre en la crianza? ¿Es beneficiosa dicha figura? ¿Qué actividades concretas realiza el padre en relación a la crianza? ¿La matrona ayuda desde su perspectiva profesional a que se desarrolle esa figura?

Es por lo que nos planteamos los siguientes objetivos.

Objetivos

- Analizar los patrones existentes de comportamiento paternal en occidente en la actualidad.

- Reivindicar la figura del padre como eje fundamental en la crianza de su hijo/a.

- Valorar y fomentar la visión de la matrona que gira en torno a la representación simbólica del rol del padre en las tareas relacionadas con la crianza.

\section{MATERIAL Y MÉTODO}

Propuesta de investigación de corte cualitativo. Utilizamos la investigación cualitativa debido al carácter del conocimiento del fenómeno de forma global, recogido en Salamanca A., y Martin-Crespo C. (2007) "tiende a ser holista, ya que se esfuerza por comprender la totalidad del fenómeno de interés". Centrándonos en el análisis de los patrones sociales objeto de este estudio, pretendemos “(...) construir un modelo de lo que se intuye en el ambiente social" (p.2) tal y como defiende este tipo de investigación.
Los instrumentos propuestos serán:

- Revisión bibliográfica en bases de datos: CUIDEN, ISOC, Cochrane.

- Entrevistas en profundidad a los sujetos de estudio.

- Grupos de discusión a las matronas como figura profesional más representativa y que está directamente implicada con la salud reproductiva de la mujer y del cuidado del recién nacido.

Abogamos por la entrevista en profundidad como técnica de elección en este estudio entendida según Blasco T., y Otero L. (2008) como "los reiterados encuentros, cara a cara, entre el investigador y los informantes, encuentros éstos dirigidos hacia la comprensión de las perspectivas o situaciones, tal como las expresan con sus propias palabra” (p.2)

El objeto de estudio será:

- Los padres (hombres) noveles.

- Matronas.

El estudio de investigación propuesto será llevado a cabo por dos residentes de matrón de la Unidad Docente de Matronas de la Universidad de Murcia y del Servicio Murciano de Salud. El contexto en el que se realizará el estudió será el área I de Salud de la Región de Murcia, en Atención Primaria de Salud, zona básica Murcia/El Palmar.

Se llevará a la práctica con sujetos voluntarios durante el primer semestre del año 2012, previa petición escrita a los participantes.

Consideraciones éticas. Se pedirán los permisos oportunos a la institución sanitaria y al comité de ética, así como a los participantes del estudio mediante el consentimiento informado. 


\section{BIBLIOGRAFÍA}

- Abril, P., y Romero, A. (2005). Masculinidad y trabajo. Las empresas con políticas de género y sus consecuencias sobre la masculinidad. Sociología del Trabajo, 55, 3-26.

- Alberdi, I. (1999). La nueva familia española. Barcelona: Taurus.

- Alberdi, I., y Escario, P. (2007). Los hombres jóvenes y la paternidad. Bilbao: Fundación BBVA.

- Álvarez, C. (2005). Múltiples maternidades y la insoportable levedad de la paternidad en reproducción humana asistida. Revista de Antropología Social, 15, 411-455.

- Blasco T., y Otero L. (2008). Técnicas conversacionales para la recogida de datos en investigación cualitativa: La entrevista (I). Revista Nure de Investigación, 33, 1-5.

- Branchs, M.A. (1999). Género masculino: buscando al padre en la literatura. Revista Venezolana de Estudios de la Mujer, 4 (11), 37-43.

- Bock, G., y Thane, P. (1996). Maternidad y políticas de género. La mujer en los estados de bienestar europeos, 1880-1950. Valencia: Ediciones Cátedra.

- BOE n.242, 7 octubre 2009.

- Bonino, L. (2003). Las nuevas paternidades. Cuadernos de trabajo social, 16, 171-182.

- Burns, N., y Grove S.K. (2011). Investigación en Enfermería. Barcelona: EDIDE, S.L.

- Calderón E., y Morales A. (2007). La madre en la antigüedad: literatura, sociedad y religión. Madrid: Signifer Libros.

- Foucault, M. (1998). El sujeto y el poder. Revista Mexicana de Sociología, 50 (3), 3-20.

- Gilmore, D.D. (1994). Hacerse hombre: concepciones culturales de la masculinidad. Barcelona: Paidós.

- Héritier, F. (2003). Masculino/Femenino. El pensamiento de la diferencia. Barcelona: Editorial Ariel.

- Hidalgo, M.V. y Palacios J.(1996). Apoyo a las familias durante la transición a la paternidad: evaluación de un programa de educación de padres. [Abstract]. C \& E Cultura y educación, (4), 71-84

- Jiménez, A.B. (2004). La paternidad en entredicho. Recuperado de http://www.ugr.es/ pwlac/G20_19AnaBelen_ Jimenez_Godoy.html

- Jociles, M.I., y Rivas, A.M. (2010). ¿Es la ausencia del padre un problema? La disociación de los roles paternos entre las madres solteras por elección. Recuperado de http://www.ugr.es/ pwlac/G26_04Isabel_Jociles-AnaMaria_Rivas.html

- Martine, S. (1992). Antropología histórica de la familia. Madrid: Taurus Universitari

- Marriner, A. (2008). Modelos y teorías en enfermería. Madrid: Elsevier Science

- Moncó, B., y Rivas, A.M. (2007). La importancia de "nombrar". El uso de la terminología de parentesco en las familias reconstituidas. Recuperado de: http://www. ugr.es/ pwlac/G23_23Beatriz_Monco_y_AnaMaria_ Rivas.html

- Nash, M. (1984). Presencia y protagonismo. Aspectos de la historia de la mujer. Barcelona: Ediciones del Serbal

- Paterna, C., Martínez C. y Rodes, J. (2005).Creencia de los hombres sobre lo que significa ser padre. Interamerican Journal of Psychology, 32(2), 275-284

- Perdiguero, E., y Comelles, J.M. (2000). Medicina y cultura. Estudios entre la antropología y la medicina. Barcelona: Edicions Bellaterra 2000.

- Real Academia Española. (Vigesimo segunda edición) (2001). Diccionario de la lengua española. Recuperado de: www.rae.es

- Requejo, A. (1979). Perspectivas educativas de la antropología sociopsicoanalitica. Recuperado de: http://ddd. uab.cat/pub/enrahonar/0211402Xn5-6p163.pdf

- Salamanca A., y Martin-Crespo C. (2007). El diseño de la investigación cualitativa. Revista Nure Investigación, $26,1-6$.

- Sefton, A.P. (2006). Paternidades de las culturas contemporáneas. Revista de estudios de género. La ventana, 23, 37-69.

- Siles, J. (2011). Historia de la Enfermería. Alicante: DAE S.L. Universidad de Alicante.

- Stuart, J. (1999). La esclavitud femenina. Recuperado de:http://www.cervantesvirtual.com/servlet/SirveObras/02589516444614584232268/index.htm

- Torns, T. (1995). Mercado de trabajo y desigualdades de género. Cuadernos de Relaciones Laborales, 6, 81-92.

- Vicuña, J. y Reyes, M.V. (2002). El Rol del Padre y su Influencia en los Hijos. Fundación Chile Unido, 64, 1-2. 